\title{
非晶 $\mathrm{Fe}_{78} \mathrm{~B}_{13} \mathrm{Si}_{9} / \mathrm{Si}$ 调制膜的 CEMS 研究
}

\author{
毕耤云 李卫东 梅良模
}

(山东大学物理系, 济南)

\section{关链词调制膜、转换电子的 Mössbauer 谱、原子扩散、过渡层}

我们用践射技术制得了非晶 $\mathrm{Fe}_{78} \mathrm{~B}_{13} \mathrm{Si}_{9} / \mathrm{Si}$ 调制膜，并用转换电子的 Mössbauer 谱技术 (CEMS) 研究了它们的微观结构. 实验结果表明, Fe 原子和 $\mathrm{Si}$ 原子在界面处的互扩散所形 成的过渡层的特性是决定这种调制膜性能的主要因素.

\section{一、引甾}

近来人们对成份调制膜的研究产生了浓厚的兴趣 ${ }^{[1]}$. 一方面是因为这种膜具有一些奇特 的物理性质; 另一方面也为制备一些自然界不存在的新型材料提供了一种新方法. 对于这种 膜的研究,过去多用二种晶格结构相近似的元素,如 $\mathrm{Cu}$ 和 $\mathrm{Ni}^{[2]}$ 制成的, 并且它们之间的相互 扩散也很小. 在本文中的调制膜是用非晶合金 $\mathrm{Fe}_{78} \mathrm{~B}_{13} \mathrm{Si}_{9}$ 和 $\mathrm{Si}$ 构成的. 用 $\mathbf{X}$ 射线的衍射技术 和转换电子的 Mössbauer 谱技术 (CEMS) 研究了这种膜的一些基本特性,揭示了 Fe 原子和 Si 原子在界面处相互扩散的重要性.

\section{二、实 验}

在实验中所用的调制膜是在 $0.1 \mathrm{~mm}$ 厚的玻俩衬底上,用双源射频谢射技术，互相交替地 沉积非晶 $\mathrm{Fe}_{78} \mathrm{~B}_{13} \mathrm{Si}_{9}$ 和 $\mathrm{Si}$ 而成的. 在沉积前的真空度 在 $2.7 \times 10^{-4} \mathrm{~Pa}$ 以下, 在浌射时的氯气压约 $2.0 \mathrm{~Pa}$. 在 戚射中祄底通水冷却. 所要求的 $\mathrm{Si}$ 层和 $\mathrm{Fe}_{78} \mathrm{~B}_{13} \mathrm{Si}_{9}$ 层 厚度通过在沉积速率保持恒定的情况下, 控制沉积的 时间来实现.

用 $\mathrm{X}$ 射线的衍射实验可以确立这种调制膜的非晶 结构, 而它的调制波长也可通过 $\mathrm{X}$ 射线的小角衍射来 确定 ${ }^{[3]}$. 图 1 示出了 $X$ 射线小角衍射所得到的结果, 由 此求出来的调制波长与预期的值基本一致. 可观察到 $n>4$ 的衍射峰, 说明样品有良好的调制结构; 主峰较 宽, 次峰较弱, 表明在界面处两种成份有混合, 没有一 个成份陡变的界面.

在室温下的转换电子的 Mössbauer 谱是用强度 约 $5 \mathrm{mCi}$ 的 ${ }^{57} \mathrm{Co} / \mathrm{Rh}$ 源, 在恒加速的 Mössbauer 谱 仪上测得的 ${ }^{[4]}$. 谱仪的速度用标准铁箔校正. 为 $\mathrm{j}$ 方

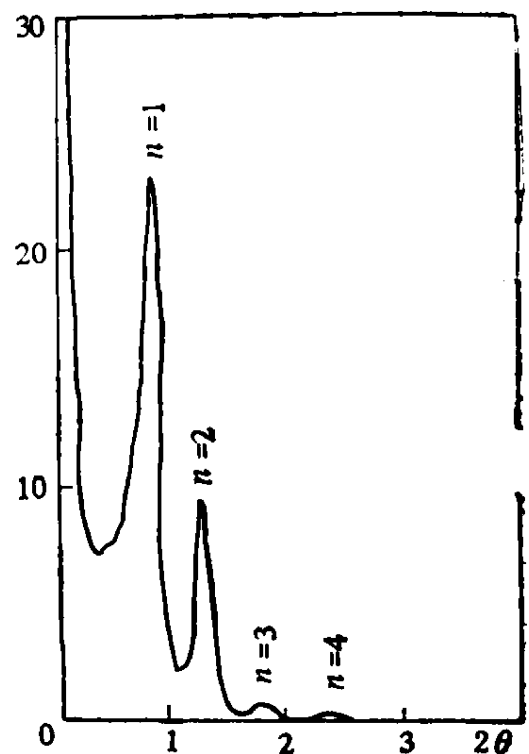

图 1 样品 (55/90) 的 X 射线小角衍射图

本文 1987 年 7 月 7 日收到.

第 19 期

科学 通报

1459 
便起见，我们用符号 $(m / n)$ 来表示被测样品的调制特性,其中 $m$ 表示在一个周期内 $\mathrm{Fe}_{73} \mathrm{~B}_{13} \mathrm{Si}$ 层的厚度 (以 $\AA$ 为单位), $n$ 表示 $S i$ 层厚. 所有样品的总周期数都是 40 . 为了比较, 我们还测 且了 $\mathrm{Fe}_{33} \mathrm{~B}_{13} \mathrm{Si}_{9}$ 单层膜(约 $2000 \AA$ ) 的 Mössbauer 谱. 对所测得的谱都用在 $\mathrm{PDP}_{11} / 34$ 计算机 系统上的程序 MOSFUN ${ }^{(3)}$ 进行拟合.考虑到在非晶材料中的谱线增宽对谱线形状的影响, 我 们用 Meisel 所提出来的方法 ${ }^{(6)}$, 用高斯和洛伦茨型的混合去拟合所测得的 Mössbauer 㙕.

\section{三、结果和讨论}

图 2 示出了在室温下所得的 Mössbauer 谱,表 1 中列出的是各种被测样品通过拟合所得 出的谱参数和由谱参数计算出来的各相之比. 在图 2 所示的二组 Mössbauer 谱中, (a)组的 $\mathrm{Fe}_{n} \mathrm{~B}_{12} \mathrm{Si}_{9}$ 层厚固定为约 $50 \AA$, (b) 组固定为约 $36 \AA$.

考查一下图 2 和表 1 便可看出，几乎所有调制膜的 Mössbauer 谱都是由两部分组成的: 一个是线间距变小,谱线加宽了的非晶六指谱,另一个是四极分裂的双线谱. 谱中这两相的相 百分比与 $\mathrm{Fe}_{n \mathrm{~B}} \mathrm{~B}_{\mathrm{i}} \mathrm{Si}_{\mathrm{g}}$ 层和 $\mathrm{Si}$ 层的厚度有关. 图 3 给出了顺磁相的百分比随 $\mathrm{Si}$ 层厚度变化的 规律 (以 $\mathrm{Fe}_{73} \mathrm{~B}_{13} \mathrm{Si}_{9}$ 层厚为参数). 可以看出, 只有当 $\mathrm{Si}$ 层的厚度大于某一临界值后才会出 现非磁性的顺磁相. 它的百分比随着 Si 层厚度的增加而增大, 直到在某一厚度下达到饱 和. 但是,顺磁相的 Mössbauer 谱参数一一四极分裂、谱线宽度和化学移,与 $\mathrm{Fe}_{73} \mathrm{~B}_{13} \mathrm{Si}_{9}$ 层和 $\mathrm{Si}$ 层的厚度无关, 是一些常量, 这表明在顺磁相中的 $\mathrm{Fe}$ 原子有基本相同的化学环境和几何结 构,并且保持不变.

表 1 Mössbauer 谱参数

\begin{tabular}{|c|c|c|c|c|c|c|}
\hline 样 品 & $\begin{array}{l}2-5 \text { 案 } \\
(\mathrm{mm} / \mathrm{s})\end{array}$ & $\begin{array}{c}2-5 \text { 知间距 } \\
(\mathrm{mm} / \mathrm{s})\end{array}$ & 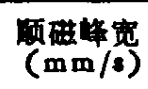 & $\begin{array}{c}\text { 顺磁化学移 } \\
(\mathrm{mm} / \mathrm{s})\end{array}$ & $\begin{array}{l}\text { 页磁分裂 } \\
(\mathrm{mm} / \mathrm{s})\end{array}$ & $\begin{array}{c}\text { 顾嘫相百 } \\
\text { 分比 }\end{array}$ \\
\hline $\mathrm{Fe}_{,}, \mathrm{B}_{1}, \mathrm{Si}$, 膜 & 0.48 & 4.91 & & & & \\
\hline$(55 / 90)$ & 0.72 & 4.60 & 0.25 & 0.24 & 0.64 & 0.220 \\
\hline$(50 / 63)$ & 0.72 & 4.50 & 0.23 & 0.22 & 0.65 & 0.206 \\
\hline$(56 / 48)$ & 0.72 & 4.50 & 0.24 & 0.23 & 0.62 & 0.181 \\
\hline$(50 / 22)$ & 0.74 & 4.45 & 0.22 & 0.24 & 0.63 & 0.062 \\
\hline$(53 / 15)$ & 0.65 & 4.55 & 0.10 & 0.24 & 0.56 & 0.013 \\
\hline$(36 / 58)$ & 0.84 & 4.20 & 0.25 & 0.20 & 0.67 & 0.336 \\
\hline$(36 / 45)$ & 0.87 & 4.15 & 0.24 & 0.20 & 0.64 & 0.315 \\
\hline$(37 / 30)$ & 0.85 & 4.20 & 0.26 & 0.20 & 0.66 & 0.260 \\
\hline$(36 / 15)$ & 0.80 & 4.25 & 0.23 & 0.21 & 0.62 & 0.120 \\
\hline
\end{tabular}

注: 表中的顺磁化学移是相对于 $\alpha-\mathrm{Fe}$.

与 $\mathrm{Fe}_{73} \mathrm{~B}_{13} \mathrm{Si}_{9}$ 单层膜相比，调制膜中的磁性分量虽然仍保持非晶合金 Mössbauer 谱的基 本特征, 但它的超精磁场降低了, 谱线增宽了. 表中所给出的 2.5 峰间距和线宽的值说明了这 一点. 另一个值得注意的事实是, 线宽和超精细场的上述变化, 只与 $\mathrm{Fe}_{78} \mathrm{~B}_{23} \mathrm{Si}_{9}$ 层的厚度有关, 不随 $\mathrm{Si}$ 层而改变.

如果我们假定: 由于扩散, 在界面处形成了 $\mathrm{Fe}$ 原子浓度从 $\mathrm{Fe}_{{ }_{3}} \mathrm{~B}_{13} \mathrm{Si}$ 到 $\mathrm{Si}$ 逐渐被稀释的 过渡层, 并且在 $\mathrm{Fe}_{7 \mathrm{~B}} \mathrm{~B}_{13} \mathrm{Si}$ 和 $\mathrm{Si}$ 都很厚的情况下, 它的厚度和 $\mathrm{Fe}$ 原子的分布只依赖于沉积时的 祄底温度,就能解释 Mössbauer 谱的上述特性. 当过渡层中的 $\mathrm{Fe}$ 原子浓度被 $\mathrm{Si}$ 稀释到一定 程度(约 0.4), 由于近邻大都是一些非磁性的 Si 原子, 交换作用被削弱了, 于是就出现了顺磁 


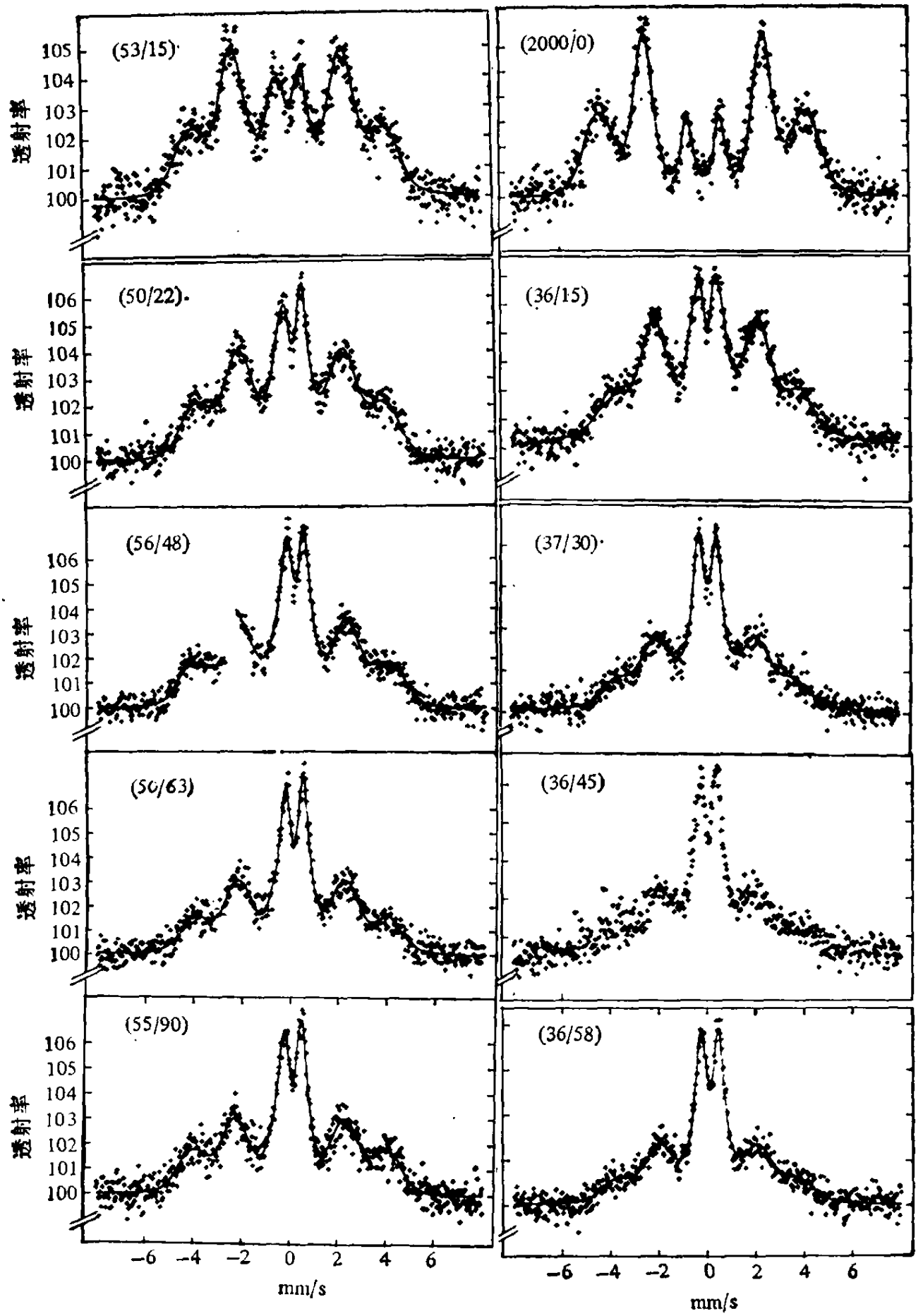

(a)

(b)

图 2 调制膜的转换电子 Mössbauer 谱

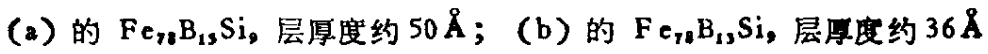

相. 显然这些顺磁性的 $\mathrm{Fe}$ 原子位于过渡区域中的 $\mathrm{Si}$ 层一侧. 如果 $\mathrm{Si}$ 层很薄, 由于从两侧向 $\mathrm{Si}$ 中扩散的 $\mathrm{Fe}$ 原子相叠加的结果, 使 $\mathrm{Si}$ 中的 $\mathrm{Fe}$ 原子浓度都大于出现顺磁相的临界值, 故顺 磁峰消失, 如图 3 所示. 当 $\mathrm{Si}$ 层的厚度大于出现顺磁相的临界厚度而小于 $\mathrm{Fe}$ 原子向 $\mathrm{Si}$ 中扩 散的最大距离的二倍时, 顺磁相的百分比将随 Si 层厚度的增加而增大, 直到饱和. 由顺磁相 饱和时的 $\mathrm{Si}$ 层厚度可求出 $\mathrm{Fe}$ 原子扩散的最大距离. 对于两相之比与 $\mathrm{Fe}_{78} \mathrm{~B}_{13} \mathrm{Si}$ 层厚度的关 


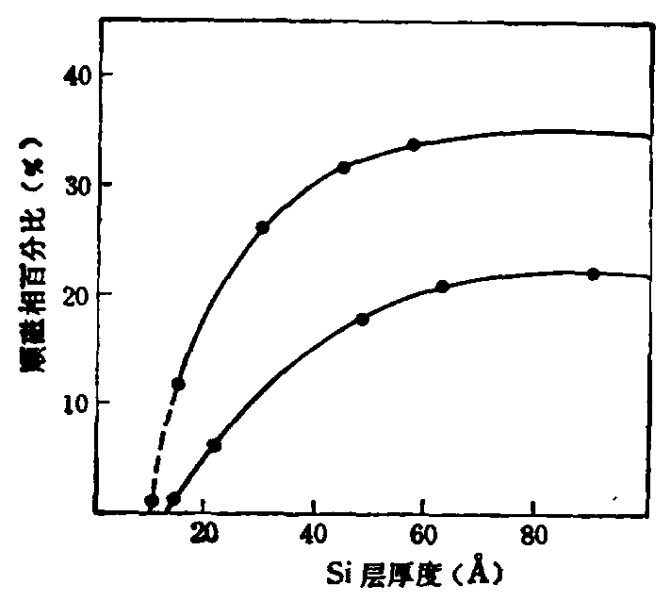

图 3 磁相的百分比与 Si 层厚度的关系

国中还示出了两条曲线所对应的 $\mathrm{Fe}_{23} \mathrm{~B}_{13} \mathrm{Si}$, 层示度, 上线: 36, 下线: 50

系,也可用类似方式解释.

用上述模型来说明 Mössbauer 谱参数的变化是十分简单的. 在过渡层中的磁性相,成份 是不均匀的,与均匀的非晶材料 $\mathrm{Fe}_{78} \mathrm{~B}_{13} \mathrm{Si}_{9}$ 相比,超精细场偏低,分布也变宽, 因而所测得的谱 线宽增大,间距变小. 在 $\mathrm{Fe}_{73} \mathrm{~B}_{13} \mathrm{Si}$ 层厚度一定的条件下, 只要 $\mathrm{Si}$ 层厚超过了临界值, 由于在 过渡层中的磁性相已饱和,故 $\mathrm{Si}$ 层厚度的增加, 不会引起六指谱参数的新变化. 当 $\mathrm{Fe}_{73} \mathrm{~B}_{13} \mathrm{Si}$ 层改变时, 不但改变了过渡层的结构, 而且也改变了过渡层中的磁性相在总磁性相中的百分 比, 因所测得谱参数理应不同. 至于顺磁相谱参数的值: 化学移 $\delta=0.23 \mathrm{~mm} / \mathrm{s}$ (相对于 $\alpha-$ $\mathrm{Fe}$ )，四极分裂 $\mathrm{QS} \doteq 0.65 \mathrm{~mm} / \mathrm{s}$ 和线宽 $\Gamma \doteq 0.25 \mathrm{~mm} / \mathrm{s}$ ，与 Oswald ${ }^{(7)}$ 所报道的非晶 $\mathrm{FeSi}$ 膜 相接近. 因此, 认为在过渡层中的非磁性相有类似于非晶 $\mathrm{FeSi}$ 的结构是合理的.

\section{四、结论}

用 CEMS 技术所测得的非晶 $\mathrm{Fe}_{78} \mathrm{~B}_{13} \mathrm{Si}_{9} / \mathrm{Si}$ 成份调制膜的 Mössbauer 谱, 可用在界面处 由于原子互扩散所形成的过渡层模型来解释。

\section{今 辛献}

: [ 1 ] Schuller, I. K. and Falco, C. M., VLSI ilectronics, Microstructure Science, 4(1983), 183.

[ 2 ] Zheng, J. Q., Falco, C. M., Ketterson, and Schuller, 1. K., Appl. Phys. Lett, 38(1981), 427.

[ 3 ] Noboru, Sato, J. Appl. Phys, 59(1986), 2514.

[4] 沈永杭、思云,山东大学学报(自然科学版), 21(1986),4: 29.

[ 5 ] Fricke, B., Laborberieht Nr. 27, Institut f. tech. Kernphysik der TH Darmstadt.

[6] Meisel, W., Experimenselle Technik der Physik. 19 (1971), 23.

[ 7 ] Oswald, R. S., Ron, M. and Ohring, M., Solid Szase Commun, 26(1978), 883. 